\title{
Unusual Contents of Inguinal Hernia Sac. An Approach to Management
}

\author{
Norman Oneil Machado, Nikita Neha Machado \\ Department of Surgery, Sultan Qaboos University Hospital, \\ Muscat, Oman \\ E-mail: oneilnorman@gmail.com \\ Received February 18, 2011; revised May 31, 2011; accepted June 10, 2011
}

\begin{abstract}
Background: unusual contents of hernia sac are uncommon, but are likely to be encountered by a surgeon in his career due to the frequency of hernia repair. The aim of this study, is to present our experience of unusual contents in inguinal hernia sac, discuss its management and review the relevant literature with regards to others experience. Patients and methods: retrospective study of 662 patients who underwent inguinal hernia repair over an 8 year period from 2000 to 2008 was carried out. Results: seven patients presented with unusual contents in inguinal hernia sac; an incidence of $1.05 \%$. Three of them had vermiform appendix, with acute appendicitis (Amyand's Hernia) noted in one of them. All patients underwent appendicectomy with repair of hernia, with mesh being employed only in patients with normal appendix. In 2 cases urinary bladder had herniated and there was one case each of ovarian cyst and fallopian tube with ovary as its content. In all these patients hernia repair was carried out after carefully reducing the contents. Conclusion: unusual contents of hernia may pose a surgical dilemma during hernia repair even to an experienced surgeon. Although rare, a hernia may contain vermiform appendix and exceptionally it may be acutely inflamed. Tubal and ovarian herniation in an inguinal hernia may be found in adult and perimenopausal women, though the incidence is reported to be more common in children. Urinary bladder herniation occurs with similar incidence as tubo ovarian hernia; however it requires special attention because of the risk of iatrogenic bladder injury during inguinal dissection. Though appendix as a content is dealt with by appendicectomy followed by hernioplasty, every effort should be made to preserve other organs found in the hernia sac to achieve an uneventful postoperative period.
\end{abstract}

Keywords: Inguinal Hernia, Amyand’s Hernia, Appendix, Ovarian Cyst, Hernia Repair

\section{Introduction}

Inguinal hernia repair is one of the commonest operations in surgical practice. However, when a surgeon encounters unusual content it could pose a difficulty in its management [1,2]. The often encountered unusual contents are appendix, ovary, fallopian tubes and urinary bladder [1,2].The presence of unusual contents of inguinal hernia has been reported exceptionally in the literature and most studies are low volume case reports. In this study we aim to present our experience of unusual contents and the approach to its management and review the relevant literature.

\subsection{Materials and Methods}

This is a retrospective study of 662 patients who underwent inguinal hernia repair at our institution between January 2000 to December 2008. The patient's hospital records were analyzed for the unusual content of hernia sac, including vermiform appendix, ovary and fallopian tubes, and urinary bladder. The demographic details, the type of hernia, surgical procedure and intraoperative findings and operative complications were recorded

\subsection{Results}

Of the 662 patients, 438 were indirect hernias and 224 
were direct hernias. There were 654 male patients and 8 female patients. The organs found in the hernia sac were vermiform appendix, urinary bladder, ovaries and fallopian tubes and ovarian cyst.

\section{Vermiform Appendix and Acute Appendicitis}

The inguinal sac was found to contain a normal appendix in 2 cases and an inflamed appendix (Amyand's Hernia) in one patient. The mean age of patients was 28 years (4 48 years). All patients were males. In 2 patients with normal appendix the hernia was on the right side (Figure 1) and in the patient with inflamed appendix it was on the left side. All patients underwent appendicectomy. In patients with normal appendix this was followed by tension free hernia repair using a vicrylprolene mesh. In the patient with acute appendicitis, following appendicectomy a thorough wash of the sac was carried out and it was then ligated and a repair without a mesh was carried out.

\section{Vescio Inguinal Hernia}

Urinary bladder was found in the hernia sac of 2 patients with direct hernias. Both the patients were males and their mean age was 60 years (52 \& 68). One of these patients had benign prostatic hypertrophy which was treated 8 weeks prior to hernia repair with transurethral resection of prostate. Both patients underwent tension free repair of the hernia using vicrylprolene mesh after careful reduction of the urinary bladder from the sac.

\section{Ovary and Fallopian Tube/Ovarian Cyst}

Ovary with fallopian tube was the content in one patient

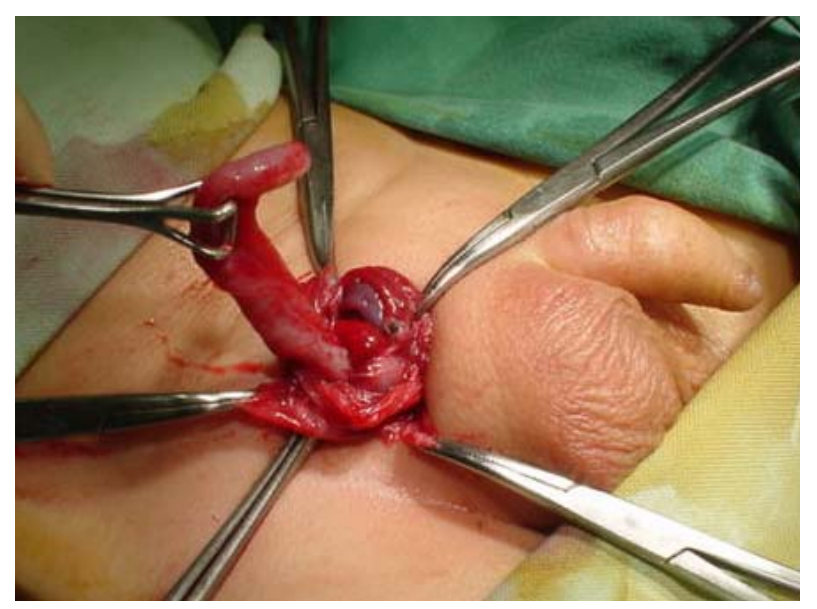

Figure 1. Normal appendix as a content of right inguinal hernia in a 4 year old boy. who was a 5 year old girl. The contents were healthy and had no torsion and no associated genital malformation. The patient underwent reduction of the contents followed by ligation of the sac. The ovarian cyst was seen in a 48 year old lady who presented with left iliac fossa pain of 6 months duration associated with inguinolabial swelling (Figure 2(a)). Following the confirmation on MRI scan (Figure 2(b)) the patient underwent laparoscopic excision of the ovarian cyst and transabdominal preperitoneal mesh repair.

There were no complications noted in any of these patients, nor recurrence.

\section{Discussion}

Inguinal hernia is a common surgical problem which

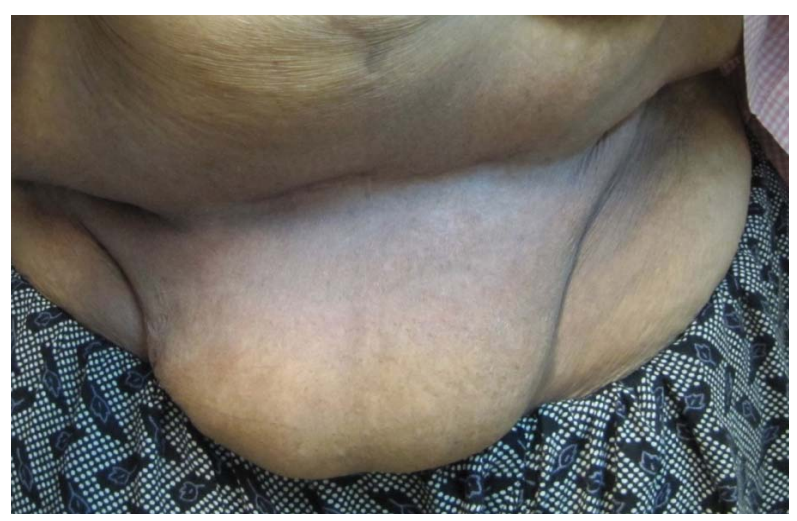

(a)

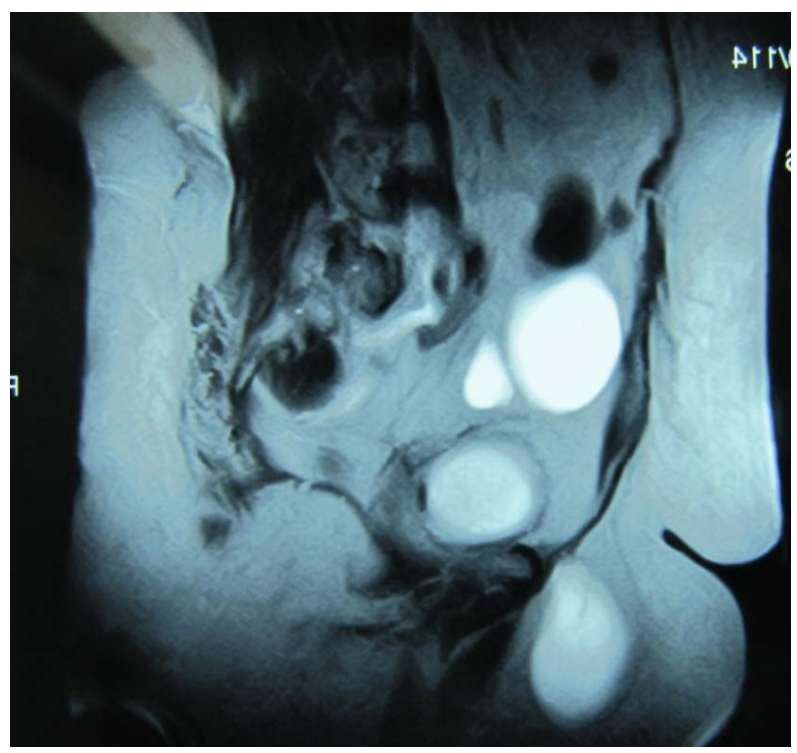

(b)

Figure 2. (a) Prominent inguinal labial swelling due to herniation of ovarian cyst into left hernia sac; (b) MRI- Sagittal view revealing ovarian cyst in the pelvis and its extension into the inguinal region. 
may sometime surprise the surgeon with its unusual content $[1,2]$. This may pose problems in management due to its surprise element. Almost all intra abdominal contents including stomach and their pathologies have been reported to have been found in the hernia sac $[3,4]$. Moreover, acute conditions including perforated diverticular abscess have also been reported to track into the inguinal canal mimicking a strangulated inguinal hernia [5]. Patients with no evidence of bowel obstruction clinically and radiologically, presenting with painful inguinal swelling have a risk of significant extra-abdominal or intra-abdominal disease processes [5]. An infected hip prosthesis abscess, a subcutaneous fungal abscess, pancreatic psuedocyst [5], leaking abdominal aortic aneurysms [6] and peritonitis have presented as an atypical inguinal hernia [7].

Although definite diagnosis of inguinal hernia is often established by the cough impulse and the ability to reduce the swelling into the abdominal cavity, clinician may face difficulties in the diagnosis of non reducible masses. The differential diagnosis could include inguinal lymphadenopathy, spermatic cord cysts, undescended testis, lipoma. While sonographic examination of this region is usually sufficient to confirm the diagnosis, CT scan or MRI could be better in anatomical delineation of its content.

The presence of a normal vermiform appendix in an inguinal hernia sac is uncommon with a reported incidence of $0.6 \%$ [8] to $1 \%$ [9] of inguinal hernias. They are usually on the right side and often seen in males. It has generally been associated with large indirect inguinoscrotal hernias but occasionally direct hernias may also contain them $[1,8]$. Acute appendicitis within an inguinal hernia accounts for $0.1 \%$ of all cases [1]. They are invariably on the right side and exceptionally on left side as in one of our cases [10]. Inflammation of the appendix is attributed to the external compression of the appendix at the neck of the hernia. The inflammatory state of the appendix determines the surgical approach and the type of hernia repair. In the case of acute appendicitis, following appendicectomy the hernia repair is performed without a synthetic mesh, due to the ongoing infective and inflammatory process [2]. While there are some who carry out appendicectomy, in the case of normal appendix incidentally found within the sac [1], there are others who feel that the addition of prophylactic appendicectomy to the hernia repair is not advisable [2]. Those who do not favour it, feel that appendicectomy adds to the possibility of infection to an otherwise clean procedure [2]. Superficial wound infection adds to the morbidity while deep infection may contribute to recurrence. In addition, the surgical effort to visualize the entire appendix by enlarging the hernia defect or distending the neck of the sac may increase the possibility of recurrence by weakening of the anatomic buttress around the hernia sac $[2,10]$. Those in favour of appendicectomy however, feel that the partly traumatized appendix, due to its handling is better dealt with by an appendicectomy, with outmost care taken to prevent contamination.

Entrapment of adnexae in an indirect inguinal hernia is rare in adult women. Most reported cases concern the paediatric population in whom ovaries and tubal herniation have long been associated as unusual contents [11]. The reported incidence of its occurrence is $71 \%$ in children under 5 years [12] and 30\% in adolescents or women in reproductive age group [12] and 2.9\% exclusively in adults [1]. Embryogenic derangements are associated with this condition. During embryogenesis the gubernaculums and broad ligaments suspend the ovary and prevent its descent through the canal of Nuck (process vaginalis peritonei) to the base of the labium major [2]. The canal of Nuck is obliterated by the $8^{\text {th }}$ week of fetal life and the ovary is then suspended between the cornu of the uterus and the internal ring. If it remains patent the ovary and the fallopian tube may be forced through the canal to a congenital hernia sac. When ovary and fallopian tube form the contents of the inguinal hernia sac, they are often associated with anomalies in the development of the genital tract such as vaginal atresia, bicornuate uterus and renal anomalies [1,2,11,12]. These patients are treated with reduction of the content provided there is no ovarian or tubal abnormality, the blood supply is not impaired and there is no evidence of salpingitis [1,2]. Reduction of the content is followed by high ligation of the hernia sac, closure of internal ring and re-enforcement of the posterior wall with a mesh in patients older than 20 years of age [1,2,12]. The presentation of the ovarian cyst as an inguinolabial swelling however is extremely rare and has been reported only once before in English literature [13] .The ovarian cyst rarely herniates into the canal of Nuck as it is usually obliterated by birth. Moreover even if the canal were to be patent, the ovarian cyst would find it easier to expand within the pelvis rather than enter into a narrow inguinal canal.

The incidence of inguinal hernia containing urinary bladder is $0.36 \%$ [1]. Herniation of bladder is often asymptomatic and only a small percentage of them are diagnosed preoperatively [14,15]. Even though most of them are associated with direct hernias [15] there are reports of its occurrence in indirect hernias [1,14]. Male sex, obstructive urinary symptoms, older age group and obesity are some of the risk factors [1]. There is a potential risk of injury to the bladder (28.6\%), particularly when it is incarcerated in an indirect inguinal hernia [1]. The injury, if not detected on table, should be suspected 
to have occurred if there is unexplained haematuria in the postoperative period.

\section{Conclusions}

Unusual contents of inguinal hernia sac are rare, but are likely to be encountered in one's surgical career during hernia repair, as it is one of the most commonly performed surgeries. The commonest unusual contents include appendix, fallopian tubes and urinary bladder which are usually dealt with by reduction of the content and hernioplasty using a mesh. However, when appendix is the content, appendicectomy is often carried out with mesh placement being avoided only in the presence of acute appendicitis. In most other instances, every effort should be made to preserve the organ found in the hernia sac to ensure an uneventful postoperative period. Unusual contents may pose special challenges even to an experienced surgeon because of its surprise element. Being aware of this possibility, along with the appropriate management would ensure better outcome in these patients.

\section{References}

[1] A. Gurer, M. Ozdogan, N. Ozlem, A. Yildirim, H. Kulacoglu and R. Aydin, "Uncommon Content in Groin hernia Sac,” Hernia, Vol. 10, No. 2, 2006, pp. 152-155. doi:10.1007/s10029-005-0036-4

[2] K. Ballas, T. H. Kontoulis, C. H. Skouras, A. Triantafyllou, N. Symeonidis, T. H. Pavlidis, et al., "Unusual Findings in Inguinal Hernia Surgery. Report of 6 Rare Cases," Hippokratia, Vol. 13, No. 3, 2009, pp. 169-171.

[3] C. E. R. Gibbons, A. K. Malhotra and M. H. Harvey, "Inguinal Hernia an Unusual Case of Gastric Outlet Obstruction,” British Journal of Hospital Medicine, Vol. 52, 1994, pp. 360-361.

[4] M. T. Oruc, B. Kulah, B. Saylam, M. Moran, I. Albayrak and F. Coskun, "An Unusual Presentation of Metastatic Gastric Cancer Found during Inguinal Hernia Repair: Case Report and Review of Literature. Hernia, Vol. 6, No. 2, 2002, pp. 88-90. doi:10.1007/s10029-002-0063-3
[5] S. I. H. Andrabi, A. Pitale and A. A.S. El-Hakeem, "Diverticular Abscess Presenting as a Strangulated Inguinal Hernia: Case Report and Review of the Literature," Ulster Medical Journal, Vol. 76, No. 2, 2007, pp. 107-108.

[6] A. M. Abulafi, W. M. Mee and B. J. Pardy, "Leaking Abdominal Aortic Aneurysm Presenting as an Inguinal Mass,” European Journal of Vascular Surgery, Vol. 5, No. 6, 1991, pp. 695-696. doi:10.1016/S0950-821X(05)80910-7

[7] D. P. Sellu, "Pus in Groin Hernia Sacs: A Complication of Non-Generalised Peritonitis," British Journal of Hospital Medicine, Vol. 41, No. 5, 1987, pp. 759-760.

[8] C. D’Alia, M. G. Lo Schiavo, A. Tonante, F. Taranto, E. Gagliano, L. Bonanno, et al., "Amyand's Hernia: Case Report and Review of the Literature,” Hernia, Vol. 7, No. 2, 2003, pp. 89-91.

[9] W. E. G. Thomas, K. D. J. Vowles and R. C. N. Williamson, “Appendicitis in External Herniae," Annals of The Royal College of Surgeons of England, Vol. 64, 1982, pp. 121-122.

[10] R. Solecki, A. Matyja and W. Milanowski, “Amyand's Hernia: A Report of 2 Cases,” Hernia, Vol. 7, 2003, pp. 50-51.

[11] V. Mayer and F. G. Templeton, "Inguinal Ectopia of the Ovary and Fallopian Tube. Review of the Literature and Report of the Case of an Infant," Archives of Surgery, Vol. 43, No. 3, 1941, pp. 397-408.

[12] H. P. van Heesewijk, F. W. Smith, M. A. Heitbrink and F. P. Kok, "Herniation of an Ovarian Cyst Through the Inguinal Canal: Diagnosis with CT," American Journal of Roentgenology, Vol. 154, No. 1, 1990, pp. 202-203.

[13] K. D. Bradshaw and B. R. Carr, "Ovarian and tubal inguinal hernia,” Obstetrics \& Gynecology, Vol. 68, Supplement 3, 1986, pp. 50-52.

[14] M. T. Oruc, Z. Akbulut, O. Ozozan and F. Coskun, "Urological Findings in Inguinal Hernia: A Case Report and Review of the Literature," Hernia, Vol. 8, No. 1, 2004, pp. 76-79. doi:10.1007/s10029-003-0157-6

[15] L. G. Gomella, S. M. Spires, J. M. Burton, M. D. Ram and R. C. Flanigan, "The Surgical Implications of Herniation of the Urinary Bladder,” Archives of Surgery, Vol. 120, No. 8, 1985, pp. 964-968. 